\title{
SINGULAR INTEGRALS AND MAXIMAL FUNCTIONS ASSOCIATED WITH HIGHLY MONOTONE CURVES
}

\author{
BY
}

W. C. NESTLERODE

AbSTRACT. Let $\gamma:[-1,1] \rightarrow \mathbf{R}^{n}$ be an odd curve. Set

$$
H_{\gamma} f(x)=\mathrm{PV} \int f(x-\gamma(t))(d t / t)
$$

and

$$
M_{\gamma} f(x)=\sup h^{-1} \int_{0}^{h}|f(x-\gamma(t))| d t .
$$

We introduce a class of highly monotone curves in $\mathbf{R}^{n}, n>2$, for which we prove that $H_{\gamma}$ and $M_{\gamma}$ are bounded operators on $L^{2}\left(\mathbf{R}^{n}\right)$. These results are known if $\gamma$ has nonzero curvature at the origin, but there are highly monotone curves which have no curvature at the origin.

Related to this problem, we prove a generalization of van der Corput's estimate of trigonometric integrals.

Introduction. Let $\gamma:[-1,1] \rightarrow \mathbf{R}^{n}$ be an odd continuous curve. For a test function $f$ on $\mathbf{R}^{n}$ we define the "Hilbert transform along $\gamma$ " of $f$ by

$$
\left(H_{\gamma} f\right)(x)=\mathrm{PV} \int_{-1}^{1} f(x-\gamma(t)) \frac{d t}{t}
$$

and the "maximal function along $\gamma$ " of $f$ by

$$
\left(M_{\gamma} f\right)(x)=\sup _{0<\varepsilon<1} \frac{1}{\varepsilon} \int_{0}^{\varepsilon}|f(x-\gamma(t))| d t .
$$

We seek conditions on $\gamma$ which guarantee either of the estimates

(1) for each $f \in L^{p}\left(\mathbf{R}^{n}\right),\left\|H_{\gamma} f\right\|_{p} \leqslant C_{\gamma}\|f\|_{p}$, or

(2) for each $f \in L^{p}\left(\mathbf{R}^{n}\right),\left\|M_{\gamma} f\right\|_{p} \leqslant C_{\gamma}\|f\|_{p}$,

for some $p, 1<p<\infty$.

The operators $H_{\gamma}$ arise when one applies the method of rotations to nonisotropic Calderón-Zygmund operators. Thus estimates of $H_{\gamma}$ lead to estimates of a broad class of singular integrals. See Nagel, Rivière and Wainger [1], for example.

$M_{\gamma}$ is the maximal operator naturally related to $H_{\gamma}$. The estimate (2) implies the following theorem on differentiation of integrals.

(3) If $f$ is locally in $L^{p}\left(\mathbf{R}^{n}\right)$, then

$$
\lim _{\varepsilon \rightarrow 0} \frac{1}{\varepsilon} \int_{0}^{\varepsilon} f(x-\gamma(t)) d t=f(x) \text { a.e. }
$$

Received by the editors October 28, 1980.

1980 Mathematics Subject Classification. Primary 42B20, 42B25. 
(1), (2) and (3) are false for arbitrary $C^{\infty}$ curves. See Nagel and Wainger [4, Theorem 4] and Stein and Wainger [8] for the counterexamples.

We call a $C^{\infty}$ curve $\gamma:[-1,1] \rightarrow \mathbf{R}^{n}, \gamma(0)=0$, well-curved if for some $\varepsilon>0$, $\gamma([-\varepsilon, \varepsilon])$ lies in the linear span of $\left\{\gamma^{(k)}(0): k=1,2,3, \ldots\right\}$. Stein and Wainger [8], [9] have shown that (1) and (2) hold if $\gamma$ is well-curved and $1<p<\infty$.

Let $\gamma(t)=(t, \phi(t))$ be a plane curve which satisfies

(4) $\phi$ is odd, $\phi \in C^{2}([0,1]), \phi(0)=\phi^{\prime}(0)=0$ and $\phi^{\prime \prime} \geqslant 0$ and $\phi^{\prime \prime}$ is increasing on $[0,1]$.

Note that $\phi(t)=\operatorname{sgn}(t) \exp \left(-|t|^{-1}\right)$ satisfies (4), but that $\gamma$ is not well-curved. Nagel and Wainger [4] have shown that (1) holds for $\frac{5}{3}<p<\frac{5}{2}$ and that (1) may fail if $p=2$ and $\phi^{\prime \prime}$ is not monotone. Stein and Wainger [9, p. 1292] have shown that (2) holds for $2 \leqslant p \leqslant \infty$.

In this paper we introduce a class of "highly monotone" curves in $\mathbf{R}^{n}$ which reduces to the curves in (4) when $n=2$. The precise definition of this class can be found in $\S 2$. In $\$ \S 3$ and 4 we prove the following theorems under the hypothesis that $\gamma:[-1,1] \rightarrow \mathbf{R}^{n}$ is odd, $\gamma_{1}(t)=t$ and $\gamma$ is highly monotone on $[0,1]$.

THEOREM 1. $\left\|H_{\gamma} f\right\|_{2} \leqslant C_{n}\|f\|_{2}\left(f \in L^{2}\left(\mathbf{R}^{n}\right)\right)$.

THEOREM 2. $\left\|M_{\gamma} f\right\|_{p} \leqslant C_{n p}\|f\|_{p}\left(f \in L^{p}\left(\mathbf{R}^{n}\right), 2<p \leqslant \infty\right)$.

THEOREM 3. If $f$ is locally in $L^{p}\left(\mathbf{R}^{n}\right), 2 \leqslant p \leqslant \infty$, then

$$
\lim _{\varepsilon \rightarrow 0} \frac{1}{\varepsilon} \int_{0}^{\varepsilon} f(x-\gamma(t)) d t=f(x) \text { a.e. }
$$

At this point we wish to say a few words about the proofs of Theorems 1 and 2.

Theorem 1 is equivalent to the boundedness of the Fourier multiplier

$$
m_{\gamma}(\xi)=\operatorname{PV} \int_{-1}^{1} e^{-2 \pi i \xi \cdot \gamma(t)} \frac{d t}{t}
$$

In [7] Stein and Wainger introduced the following estimate of trigonometric integrals into the study of $H_{\gamma}$.

LEMMA (VAN DER CORPUT). If $f$ is a real-valued function on $[a, b]$, if $\left|f^{(n)}(t)\right|>\lambda$ $>0$ for $a \leqslant t \leqslant b$ and if $f^{\prime}$ is monotone when $n=1$, then

$$
\left|\int_{a}^{b} e^{i f(t)} d t\right|<C_{n} \lambda^{-1 / n}
$$

This lemma has been basic in the study of $H_{\gamma}$ and $M_{\gamma}$ since then.

In $\$ 1$ we prove a generalization of van der Corput's lemma which is particularly suited to highly monotone curves. The generalization comes in replacing $f^{(n)}$ by $D^{n} f$ when $n>1$, where $D^{n}$ is a suitable differential operator of order $n$. In $\S 3$ we use this estimate to prove Theorem 1 for highly monotone curves.

To prove Theorem 2 we use the method of " $g$-functions" introduced by E. M. Stein in [5] and [6]. This technique has been used to prove many maximal theorems; for examples see Stein and Wainger [8], Nagel, Stein and Wainger [3] and Wainger [10]. 
The use of $g$-functions allows one to use the Fourier transform and reduce the maximal theorem to estimating trigonometric integrals. In $\$ 4$ we use a variant of the $g$-function in Stein and Wainger [9, p. 1292] and our generalization of van der Corput's lemma to prove Theorem 2 for highly monotone curves.

It should be noted that the first application of the Fourier transform to the study of $M_{\gamma}$ was made by Nagel, Rivière and Wainger [2] in the special case $\gamma(t)=\left(t, t^{2}\right)$.

I take this opportunity to thank my teacher and advisor, Professor Stephen Wainger, for the suggestions and encouragement he has given me in the course of this work. (I would also like to thank Professor Alexander Nagel and my fellow graduate students, Jim Vance and Dave Weinberg for many useful discussions.)

1. An estimate for trigonometric integrals. In this section, we prove a lemma which will be of use in $\S \S 3$ and 4 . Before stating the lemma we must introduce some notation.

Given a smooth function $\alpha:[a, b] \rightarrow(0, \infty)$ we define a differential operator $D_{\alpha}$ by

$$
D_{\alpha} f(t)=(f / \alpha)^{\prime}(t)
$$

If $\alpha_{1}, \ldots, \alpha_{n}$ are $n$ such functions, then we inductively define operators $D^{1}, \ldots, D^{n}$,

$$
D^{1}=D_{\alpha_{1}} ; \quad D^{k+1}=D_{\alpha_{k+1}} D^{k} \quad \text { for } 1 \leqslant k<n .
$$

LEMMA 1. Let $\alpha_{1}, \ldots, \alpha_{n}$ be positive nondecreasing functions defined on $[a, b]$ and let $\alpha_{1} \equiv 1$. Let $f$ be a real-valued function of class $C^{n}$ on $[a, b]$. If $D^{1} f=f^{\prime}$ is monotone and if $\left|D^{n} f(t)\right| \geqslant \lambda>0$ for each $t$ in $[a, b]$, then

$$
\left|\int_{a}^{b} \exp [i f(t)] d t\right| \leqslant C_{n}\left(\lambda \alpha_{1}(a) \cdots \alpha_{n}(a)\right)^{-1 / n}
$$

We note that if each $\alpha_{k} \equiv 1$, then Lemma 1 reduces to van der Corput's lemma. (See [11, Volume I, p. 197] or [9, p. 1258].)

Proof. Suppose that $n=1$. To be specific, assume that $f^{\prime}$ is nonincreasing and that $f^{\prime}(t) \geqslant \lambda$. Now

$$
\int_{a}^{b} e^{i f(t)} d t=\int_{a}^{b} \frac{d}{d t}\left\{e^{i f(t)}\right\} \frac{d t}{i f^{\prime}(t)}
$$

so integration by parts yields the estimate

$$
\left|\int_{a}^{b} e^{i f(t)} d t\right| \leqslant \frac{1}{f^{\prime}(b)}+\frac{1}{f^{\prime}(a)}+\int_{a}^{b} d\left(\frac{1}{f^{\prime}}\right)=\frac{2}{f^{\prime}(b)}<\frac{2}{\lambda} .
$$

Now assume that the lemma is true for a given $n>1$. Assume that $f^{\prime}$ is monotone and that $D^{n+1} f(t) \geqslant \lambda$ for $a \leqslant t \leqslant b$.

Set $h=D^{n} f$. Then $D^{n+1} f=\left(h / \alpha_{n+1}\right)^{\prime}$. Choose $c$ in $[a, b]$ so that $h / \alpha_{n+1}$ is positive on $(c, b)$ and $h / \alpha_{n+1}$ is negative on $(a, c)$. Such a value of $c$ exists, and is unique, since $h / \alpha_{n+1}$ is increasing. 
Write $\int_{a}^{b} e^{i f(t)} d t=\int_{a}^{c}+\int_{c}^{b}=P+Q$ and estimate $P$ and $Q$ separately. To estimate $P$ suppose that $a<u<c$. Then

$$
|P| \leqslant\left|\int_{a}^{u} e^{i f(t)} d t\right|+c-u .
$$

If $a \leqslant t \leqslant u$, then

$$
\frac{h}{\alpha_{n+1}}(t) \leqslant \frac{h}{\alpha_{n+1}}(u)=\frac{h}{\alpha_{n+1}}(c)-\int_{u}^{c}\left(\frac{h}{\alpha_{n+1}}\right)^{\prime}(s) d s \leqslant-\lambda(c-u) .
$$

Hence we have

$$
D^{n} f(t)=h(t) \leqslant-\lambda \alpha_{n+1}(t)(c-u) \leqslant-\lambda \alpha_{n+1}(a)(c-u)
$$

for $a \leqslant t \leqslant u$. By the induction hypothesis

$$
|P| \leqslant C_{n}\left(\lambda \alpha_{1}(a) \cdots \alpha_{n}(a) \alpha_{n+1}(a)(c-u)\right)^{-1 / n}+c-u
$$

for $a<u<c$. This estimate actually holds for each $u<c$ since $|P| \leqslant c-a$.

Set $c-u=\left(\lambda \alpha_{1}(a) \cdots \alpha_{n+1}(a)\right)^{-1 /(n+1)}$. Then we get

$$
|P| \leqslant\left(C_{n}+1\right)\left(\lambda \alpha_{1}(a) \cdots \alpha_{n+1}(a)\right)^{-1 /(n+1)} .
$$

The estimate of $Q$ is made in a similar manner. Q.E.D.

2. Highly monotone curves. Let $\gamma:[0, N] \rightarrow \mathbf{R}^{n}$ be a curve of class $C^{n}$ with $\gamma(0)=0$. We inductively define functions $\alpha_{1}, \ldots, \alpha_{n}$ as follows.

$$
\alpha_{1} \equiv 1 ; \quad \alpha_{k+1}=D^{k} \gamma_{k} \quad \text { for } 1<k<n .
$$

Here $D^{1}, \ldots, D^{n}$ are the differential operators associated with $\alpha_{1}, \ldots, \alpha_{n}$ as in $\S 1$. At each stage of this definition we must assume that $\alpha_{k}$ is positive on $(0, N)$ so that the operator $D^{k}$ is well defined.

We now consider the matrix $W_{\gamma}=\left[D^{k} \gamma_{j}\right]_{1<k j<n}$. It is easy to see that $W_{\gamma}$ is upper triangular:

$$
W_{\gamma}=\left[\begin{array}{cccc}
D^{1} \gamma_{1} & D^{1} \gamma_{2} & \cdots & D^{1} \gamma_{n} \\
& D^{2} \gamma_{2} & \cdots & D^{2} \gamma_{n} \\
& & \ddots & \vdots \\
& & & D^{n} \gamma_{n}
\end{array}\right] .
$$

This follows since row $k+1$ of $W_{\gamma}$ is obtained by dividing row $k$ by $D^{k} \gamma_{k}$ and differentiating.

We say that $\gamma$ is highly monotone if it has the following two properties.

(1) If $1 \leqslant k \leqslant j \leqslant n$, then $D^{k} \gamma_{j}$ is positive and nondecreasing on $(0, N)$.

(2) If $1 \leqslant k<j \leqslant n$, then $D^{k} \gamma_{j}(t)=o\left(D^{k} \gamma_{k}(t)\right)$ as $t \rightarrow 0+$.

Lemma 2. Let $\gamma:[0, N] \rightarrow \mathbf{R}^{n}$ be a highly monotone curve. If $1<k<j \leqslant n$ and $0<t<N$, then

$$
D^{k} \gamma_{j}(t) \geqslant \gamma_{j}(t) / t^{k} \alpha_{1}(t) \cdots \alpha_{k}(t)
$$


Proof. We note that if $\varphi \in C^{1}([0, N]), \varphi(0)=0$ and $\varphi^{\prime}$ is nondecreasing, then we have

$$
\varphi(t) \leqslant t \varphi^{\prime}(t)
$$

Fix $j$. We prove (i) by induction on $k$.

$$
D^{1} \gamma_{j}(t)=\gamma_{j}^{\prime}(t) \geqslant \gamma_{j}(t) / t=\gamma_{j}(t) / t \alpha_{1}(t)
$$

follows from (ii) with $\varphi=\gamma_{j}$ and the fact that $\alpha_{1} \equiv 1$.

Assume (i) for some $k, 1 \leqslant k<j$. Then we have

$$
D^{k+1} \gamma_{j}(t)=\left(\frac{D^{k} \gamma_{j}}{D^{k} \gamma_{k}}\right)^{\prime}(t) \geqslant \frac{D^{k} \gamma_{j}(t)}{t \alpha_{k+1}(t)} \geqslant \frac{\gamma_{j}(t)}{t^{k+1} \alpha_{1}(t) \cdots \alpha_{k}(t) \alpha_{k+1}(t)} .
$$

The first inequality follows from (ii) with $\varphi=D^{k} \gamma_{j} / D^{k} \gamma_{k}$ and from the fact that $\alpha_{k+1}=D^{k} \gamma_{k}$. The second inequality follows from the induction hypothesis. Q.E.D.

We conclude this section with some examples of highly monotone curves.

EXAMPLE 1. Let $\gamma(t)=\left(t^{a_{1}}, \ldots, t^{a_{n}}\right)$ for $t \geqslant 0$. Suppose that $a_{1} \geqslant 1$ and $a_{j+1}>$ $a_{j}+1$. Then $\gamma$ is highly monotone and

$$
W_{\gamma}=\left[\begin{array}{cccc}
a_{1} t^{a_{1}-1} & a_{2} t^{a_{2}-1} & \cdots & a_{n} t^{a_{n}-1} \\
& c t^{a_{2}-a_{1}-1} & \cdots & c t^{a_{n}-a_{2}-1} \\
& & \ddots & \vdots \\
& & & c t^{a_{n}-a_{n-1}-1}
\end{array}\right] .
$$

The various constants $c$ are positive and depend only on $a_{1}, \ldots, a_{n}$.

EXAMPLE 2. Let $n=2$ and let $\gamma(t)=(t, \varphi(t))$. Then $\gamma$ is highly monotone if, and only if, $\varphi(0)=0, \varphi^{\prime}(0)=0$ and $\varphi^{\prime \prime}$ is positive and nondecreasing on $(0, N)$.

$$
W_{\gamma}=\left[\begin{array}{cc}
1 & \varphi^{\prime} \\
0 & \varphi^{\prime \prime}
\end{array}\right]
$$

EXAMPLE 3. Let $\gamma(t)=\left(t, t^{a_{2} e^{-t^{-\beta_{2}}}}, \ldots, t^{a_{n}} e^{-t^{-\beta_{n}}}\right)$ for $t>0$. We assume that $a_{j+1} \geqslant a_{j}+1$ and $\beta_{n} \geqslant \beta_{n-1} \geqslant \cdots \geqslant \beta_{2}>0$. For $N$ sufficiently small $\gamma$ is highly monotone on $[0, N]$.

3. The Hilbert transform. Let $\gamma:[-N, N] \rightarrow \mathbf{R}^{n}$ be a continuous curve with $\gamma(0)=0$. For $0<\varepsilon<N$ we define the truncated Hilbert transform by

$$
H_{\varepsilon, N} f(x)=\int_{\varepsilon<|t|<N} f(x-\gamma(t)) \frac{d t}{t} .
$$

In [9, p. 1284] it is shown that $H_{\varepsilon, N} f$ is a well-defined measurable function if $f$ is locally integrable on $\mathbf{R}^{n}$.

THEOREM 1. Let $\gamma:[0, N] \rightarrow \mathbf{R}^{n}$ be a highly monotone curve with $\gamma_{1}(t)=t$. For $0 \leqslant t \leqslant N$ define $\gamma(-t)=-\gamma(t)$. There exists a constant $C_{n}$, which depends only on $n$, so that if $0<\varepsilon<N$ and $f \in L^{2}\left(\mathbf{R}^{n}\right)$, then

$$
\left\|H_{e, N} f\right\|_{2} \leqslant C_{n}\|f\|_{2} \text {. }
$$


Furthermore, for each $f \in L^{2}\left(\mathbf{R}^{n}\right), H_{N} f=\lim _{\varepsilon \rightarrow 0+} H_{e, N} f$ exists in the $L^{2}$ norm and

$$
\left\|H_{N} f\right\|_{2} \leqslant C_{n}\|f\|_{2} \text {. }
$$

The case $n=2$ of Theorem 1 is due to Nagel and Wainger [4].

Let $\gamma$ be the curve in Example 3 of $\$ 2$. Then $\gamma$ has "no curvature" at the origin, i.e. $\gamma^{(k)}(0)=0$ for $k=2,3, \ldots, \gamma([0, N])$ lies in no proper subspace of $\mathbf{R}^{n}$, but, by Theorem 1, the Hilbert transform associated with $\gamma$ is bounded on $L^{2}\left(\mathbf{R}^{n}\right)$. This is in contrast to the work of Stein and Wainger [9, p. 1261, Theorem 3(B)] and the counterexample of Nagel and Wainger [4, Theorem 4.1].

Proof of Theorem 1 . An easy computation shows that $\left(H_{e, N} f\right)^{\wedge}=m_{e, N} \hat{f}$ where denotes the Fourier transform and

$$
\begin{aligned}
m_{\varepsilon, N}(\xi) & =\int_{\varepsilon<|t|<N} \exp [-2 \pi i \xi \circ \gamma(t)] \frac{d t}{t} \\
& =-2 i \int_{\varepsilon}^{N} \sin (2 \pi \xi \circ \gamma(t)) \frac{d t}{t} .
\end{aligned}
$$

Conclusion (i) is equivalent to the estimate

$$
\left|m_{\varepsilon, N}(\xi)\right| \leqslant C_{n} \quad \text { for } \xi \in \mathbf{R}^{n} \text { and } 0<\varepsilon<N .
$$

We prove (iii) by induction on $n$. The case $n=1$ is well known.

Let $n>1$ and assume that (iii) holds for $n-1$. Let $\gamma:[-N, N] \rightarrow \mathbf{R}^{n}$ satisfy the hypotheses of Theorem 1. Set $\bar{\gamma}=\left(\gamma_{1}, \ldots, \gamma_{n-1}\right)$ so that $\gamma=\left(\bar{\gamma}, \gamma_{n}\right)$. Take $\xi \in \mathbf{R}^{n}$, $\xi=\left(\bar{\xi}, \xi_{n}\right)$. If $\xi_{n}=0$, then $\left|m_{e, N}(\xi)\right| \leqslant C_{n}$ follows from the induction hypothesis applied to $\bar{\gamma}$. So assume that $\xi_{n} \neq 0$. Define $a$ to be the solution of $\left|\xi_{n}\right| \gamma_{n}(a)=1$, $0<a<N$, if it exists. Otherwise set $a=N$. (Recall $\gamma_{n}(0)=0$ and $\gamma_{n}$ is increasing. Thus $a=N$ iff $\left|\xi_{n}\right| \gamma_{n}(N) \leqslant 1$.)

$$
\begin{aligned}
\frac{\dot{-1}}{2 i} m_{\varepsilon, N}(\xi)= & \int_{\varepsilon}^{a}\{\sin (2 \pi \xi \circ \gamma(t))-\sin (2 \pi \bar{\xi} \circ \bar{\gamma}(t))\} \frac{d t}{t} \\
& +\int_{\varepsilon}^{a} \sin (2 \pi \bar{\xi} \circ \bar{\gamma}(t)) \frac{d t}{t}+\int_{a}^{N} \sin (2 \pi \xi \circ \gamma(t)) \frac{d t}{t} \\
= & P+Q+R .
\end{aligned}
$$

$|Q| \leqslant C_{n}$ follows from the induction hypothesis.

$$
\begin{aligned}
|P| & \leqslant 2 \pi\left|\xi_{n}\right| \int_{0}^{a} \gamma_{n}(t) \frac{d t}{t} \leqslant 2 \pi\left|\xi_{n}\right| \int_{0}^{a} \gamma_{n}^{\prime}(t) d t \\
& =2 \pi\left|\xi_{n}\right| \gamma_{n}(a) \leqslant 2 \pi .
\end{aligned}
$$

If $a=N$, then $R=0$. So assume that $0<a<N$. By the Second Mean Value Theorem,

$$
R=\frac{1}{a} \int_{a}^{b} \sin (2 \pi \xi \circ \gamma(t)) d t \text { for some } b, a<b<N .
$$

Set $f(t)=\xi \circ \gamma(t)$. Then $\left|D^{n} f(t)\right|=\left|\xi_{n}\right|\left(D^{n} \gamma_{n}\right)(t)>\left|\xi_{n}\right|\left(D^{n} \gamma_{n}\right)(a)$ for $a<t \leqslant N$. So Lemma 1 will imply

$$
|R| \leqslant C_{n} a^{-1}\left(\left|\xi_{n}\right|\left(D^{n} \gamma_{n}\right)(a) \alpha_{1}(a) \cdots \alpha_{n}(a)\right)^{-1 / n}
$$


if we can divide $[a, N]$ into a bounded (in $a$ and $\xi$ ) number of subintervals on each of which $f^{\prime}$ is monotone. Applying Lemma 2 we see that

$$
\left|\xi_{n}\right| a^{n}\left(D^{n} \gamma_{n}\right)(a) \alpha_{1}(a) \cdots \alpha_{n}(a) \geqslant\left|\xi_{n}\right| \gamma_{n}(a)=1
$$

so that $|R| \leqslant C_{n}$. Thus it suffices to prove (iv) below.

(iv) $\forall \xi \in \mathbf{R}^{n}, f^{\prime \prime}(t)=\xi \circ \gamma^{\prime \prime}(t)$ has at most $n-2$ zeros in $(0, N)$.

Note that $f(t)=\xi_{1} t+\sum_{j=2}^{n} \xi_{j} \gamma_{j}(t)$ so that

$$
f^{\prime \prime}(t)=\sum_{j=2}^{n} \xi_{j} \gamma_{j}^{\prime \prime}(t) \text { and } D^{k} f(t)=\sum_{j=k}^{n} \xi_{j} D^{k} \gamma_{j}(t)
$$

for $2 \leqslant k \leqslant n . D^{n} f(t)=\xi_{n} D^{n} \gamma_{n}(t)$ has no zero in $(0, N)$. Since $D^{n} f=\left(D^{n-1} f / \alpha_{n}\right)^{\prime}$, Rolle's theorem implies that $D^{n-1} f / \alpha_{n}$ has at most one zero in $(0, N)$. But $\alpha_{N}>0$. Thus $D^{n-1} f$ has at most one zero in $(0, N)$. Repeating this argument $n-2$ times shows that $f^{\prime \prime}=D^{2} f$ has at most $n-2$ zeros in $(0, N)$. This proves (iv) and with it (i).

(ii) follows from (i) and the fact that $H_{N} f=\lim _{\varepsilon \rightarrow 0+} H_{\varepsilon, N} f$ exists in $L^{2}$ if $f \in C_{c}^{1}\left(\mathbf{R}^{n}\right)$. Q.E.D.

Let $\gamma(t)=\operatorname{sgn} t\left(|t|^{a_{1}}, \ldots,|t|^{a_{n}}\right)$ where $a_{1} \geqslant 1$ and $a_{j+1} \geqslant a_{j}+1$ for $1 \leqslant j<n$. The proof of Theorem 1(i) goes through for this $\gamma$ with a few minor changes. This is essentially the proof given by Stein and Wainger in [7].

4. The maximal function. Let $\gamma:[0, N] \rightarrow \mathbf{R}^{n}$ be a continuous curve with $\gamma(0)=0$. We define the maximal function by

$$
M f(x)=\sup _{0<\varepsilon<N} \frac{1}{\varepsilon} \int_{0}^{\varepsilon}|f(x-\gamma(t))| d t .
$$

In [9, p. 1284] it is shown that $M f$ is a well-defined measurable function if $f$ is locally integrable on $\mathbf{R}^{n}$.

THEOREM 2. Let $\gamma:[0, N] \rightarrow \mathbf{R}^{n}$ be a highly monotone curve with $\gamma_{1}(t)=t$.

(i) There is a constant $C_{n}$, which depends only on $n$, such that for each $f \in L^{2}\left(\mathbf{R}^{n}\right)$, $\|M f\|_{2} \leqslant C_{n}\|f\|_{2}$.

(ii) If $2 \leqslant p \leqslant \infty$, there is a constant $C_{n, p}$ such that for each $f \in L^{p}\left(\mathbf{R}^{n}\right)$, $\|M f\|_{p} \leqslant C_{n, p}\|f\|_{p}$.

THEOREM 3. Let $\gamma:[0, N] \rightarrow \mathbf{R}^{n}$ be a highly monotone curve with $\gamma_{1}(t)=t$. If $f$ is locally in $L^{p}\left(\mathbf{R}^{n}\right), 2 \leqslant p \leqslant \infty$, then

$$
\lim _{\varepsilon \rightarrow 0} \frac{1}{\varepsilon} \int_{0}^{\varepsilon} f(x-\gamma(t)) d t=f(x) \text { a.e. }
$$

The case $n=2$ of Theorem 2 is due to Stein and Wainger [9, p. 1292].

The curve in Example 3 of $\$ 2$ has "no curvature" at the origin, lies in no proper subspace of $\mathbf{R}^{n}$, but the $L^{2}$ differentiation theorem (Theorem 3 ) is true for this curve. This is in contrast to the work of Stein and Wainger [8].

Proof of Theorem 2. We prove (i) by induction on $n$.

If $n=1$, then $M f$ is the Hardy-Littlewood maximal function and (i) is well known in this case. 
Let $n>1$ be given and assume that (i) holds for any highly monotone curve $\bar{\gamma}$ in $\mathbf{R}^{n-1}$. Let $\gamma$ be a highly monotone curve in $\mathbf{R}^{n}$. Set $\bar{\gamma}=\left(\gamma_{1}, \ldots, \gamma_{n-1}\right)$. Then $\bar{\gamma}$ is highly monotone and $\gamma=\left(\bar{\gamma}, \gamma_{n}\right)$.

We set

$$
M_{h} f(x)=\frac{1}{h} \int_{h}^{2 h} f(x-\gamma(t)) d t
$$

and

$$
N_{h} f(x)=\frac{1}{h} \int_{h}^{2 h} \frac{1}{\gamma_{n}(h)} \int_{0}^{\gamma_{n}(h)} f\left(\bar{x}-\bar{\gamma}(t), x_{n}-s\right) d s d t .
$$

We define

$$
g(f)(x)=\left\{\int_{0}^{N / 2}\left|M_{h} f(x)-N_{h} f(x)\right|^{2} \frac{d h}{h}\right\}^{1 / 2} \quad \text { for } f \in L^{2} .
$$

The argument in Stein and Wainger $[9$, p. 1265] shows that if $f>0$, then

$$
M f(x) \leqslant C\left(g(f)(x)+\sup _{0<2 h<N} N_{h} f(x)\right) .
$$

So it suffices to prove the following two estimates.

$$
\begin{aligned}
\forall f & \in L^{2}\left(\mathbf{R}^{n}\right),\left\|\sup _{h>0}\left|N_{h} f\right|\right\|_{2} \leqslant C_{n}\|f\|_{2} \\
\forall f & \in L^{2}\left(\mathbf{R}^{n}\right), \quad\|g(f)\|_{2} \leqslant C_{n}\|f\|_{2} . \\
\int_{\mathbf{R}^{n}} \sup _{h}\left|N_{h} f(x)\right|^{2} d x & =\int_{\mathbf{R}^{1}} \int_{\mathbf{R}^{n-1}} \sup _{h}\left|N_{h} f(x)\right|^{2} d \bar{x} d x_{n} \\
& \leqslant \int_{\mathbf{R}^{1}} C_{n} \int_{\mathbf{R}^{n-1}}\left(\sup _{\varepsilon>0} \frac{1}{\varepsilon} \int_{0}^{\varepsilon}\left|f\left(\bar{x}, x_{n}-s\right)\right| d s\right)^{2} d \bar{x} d x_{n} \\
& =C_{n} \int_{\mathbf{R}^{n-1}} \int_{\mathbf{R}^{1}}\left(\sup _{\varepsilon>0} \frac{1}{\varepsilon} \int_{0}^{\varepsilon}\left|f\left(\bar{x}, x_{n}-s\right)\right| d s\right)^{2} d x_{n} d \bar{x} \\
& \leqslant C_{n} \int_{\mathbf{R}^{n-1}} \int_{\mathbf{R}^{\prime}}\left|f\left(\bar{x}, x_{n}\right)\right|^{2} d x_{n} d \bar{x} \\
& =C_{n} \int_{\mathbf{R}^{n}}|f(x)|^{2} d x .
\end{aligned}
$$

The first inequality follows from the induction hypothesis; the second inequality follows from the Hardy-Littlewood maximal theorem. Hence, only (iii) remains to be proved.

Note that $\left(M_{h} f\right)^{\wedge}=m_{h} \hat{f}$ and $\left(N_{h} f\right)^{\wedge}=n_{h} \hat{f}$, where for each $\xi=\left(\bar{\xi}, \xi_{n}\right)$ in $\mathbf{R}^{n}$,

$$
m_{h}(\xi)=\frac{1}{h} \int_{h}^{2 h} e^{-2 \pi i \xi \gamma(t)} d t
$$

and

$$
n_{h}(\xi)=\frac{1}{h \gamma_{n}(h)} \int_{h}^{2 h} \int_{0}^{\gamma_{n}(h)} e^{-2 \pi i\left(\overline{\xi_{\gamma}}(t)+\xi_{n} s\right)} d s d t
$$


Using Fubini's theorem and the Plancherel theorem

$$
\begin{aligned}
\int_{\mathbf{R}^{n}}|g(f)(x)|^{2} d x & =\int_{0}^{N / 2} \int_{\mathbf{R}^{n}}\left|M_{h} f(x)-N_{h}(x)\right|^{2} d x \frac{d h}{h} \\
& =\int_{0}^{N / 2} \int_{\mathbf{R}^{n}}\left|m_{h}(\xi)-n_{h}(\xi)\right|^{2}|\hat{f}(\xi)|^{2} d \xi \frac{d h}{h} \\
& =\int_{\mathbf{R}^{n}}\left(\int_{0}^{N / 2}\left|m_{h}(\xi)-n_{h}(\xi)\right|^{2} \frac{d h}{h}\right)|\hat{f}(\xi)|^{2} d \xi
\end{aligned}
$$

So to prove (iii) it is sufficient to prove (iv).

$$
\forall \xi \in \mathbf{R}^{n}, \quad \int_{0}^{N / 2}\left|m_{h}(\xi)-n_{h}(\xi)\right|^{2} \frac{d h}{h} \leqslant C_{n} .
$$

Define $a$ to be the solution of $\gamma_{n}(2 a)=1 /\left|\xi_{n}\right|$. Write the integral in (iv) as $\int_{0}^{a}+\int_{a}^{2 a}+\int_{2 a}^{N / 2}$.

$$
\int_{a}^{2 a}\left|m_{h}(\xi)-n_{h}(\xi)\right|^{2} \frac{d h}{h} \leqslant 4 \int_{a}^{2 a} \frac{d h}{h}=4 \log 2
$$

since $\left|m_{h}(\xi)\right| \leqslant 1$ and $\left|n_{h}(\xi)\right| \leqslant 1$.

$$
\begin{aligned}
\left|m_{h}(\xi)-n_{h}(\xi)\right| & \leqslant \frac{1}{h \gamma_{n}(h)} \int_{h}^{2 h} \int_{0}^{\gamma_{n}(h)}\left|e^{-2 \pi i \xi_{n} \gamma_{n}(t)}-e^{-2 \pi i \xi_{n} s}\right| d s d t \\
& \leqslant \frac{2 \pi\left|\xi_{n}\right|}{h \gamma_{n}(h)} \int_{h}^{2 h} \int_{0}^{\gamma_{n}(h)}\left(\gamma_{n}(t)-s\right) d s d t \leqslant 2 \pi\left|\xi_{n}\right| \gamma_{n}(2 h)
\end{aligned}
$$

so that

$$
\begin{gathered}
\int_{0}^{a}\left|m_{h}(\xi)-n_{h}(\xi)\right|^{2} \frac{d h}{h} \\
\leqslant 4 \pi^{2}\left|\xi_{n}\right|^{2} \int_{0}^{a} \gamma_{n}(2 h)^{2} \frac{d h}{h} \\
\leqslant 4 \pi^{2}\left|\xi_{n}\right|^{2} \gamma_{n}(2 a) \int_{0}^{2 a} \frac{\gamma_{n}(t)}{t} d t \\
\leqslant 4 \pi^{2}\left|\xi_{n}\right|^{2} \gamma_{n}(2 a) \int_{0}^{2 a} \gamma_{n}^{\prime}(t) d t \\
=4 \pi^{2}\left|\xi_{n}\right|^{2} \gamma_{n}(2 a)^{2}=4 \pi^{2} \\
\int_{2 a}^{N / 2}\left|m_{h}(\xi)-n_{h}(\xi)\right|^{2} \frac{d h}{h} \leqslant 2 \int_{2 a}^{N / 2}\left|m_{h}(\xi)\right|^{2} \frac{d h}{h}+2 \int_{2 a}^{N / 2}\left|n_{h}(\xi)\right|^{2} \frac{d h}{h}
\end{gathered}
$$

and we estimate each of the last integrals separately.

Making use of Lemma 1 and the argument in the proof of Theorem 1 we see that

$$
\left|m_{h}(\xi)\right| \leqslant C_{n}\left(\left|\xi_{n}\right|\left(D^{n} \gamma_{n}\right)(h) \alpha_{1}(h) \cdots \alpha_{n}(h)\right)^{-1 / n} h^{-1} .
$$

Squaring and integrating shows that

$$
\begin{aligned}
\int_{2 a}^{N / 2}\left|m_{h}(\xi)\right|^{2} \frac{d h}{h} & \leqslant C_{n}\left(\left|\xi_{n}\right|\left(D^{n} \gamma_{n}\right)(2 a) \alpha_{1}(2 a) \cdots \alpha_{n}(2 a)\right)^{-2 / n} \int_{2 a}^{\infty} \frac{d h}{h^{3}} \\
& =C_{n}\left(\left|\xi_{n}\right|(2 a)^{n} D^{n} \gamma_{n}(2 a) \alpha_{1}(2 a) \cdots \alpha_{n}(2 a)\right)^{-2 / n} \\
& \leqslant C_{n}\left(\left|\xi_{n}\right| \gamma_{n}(2 a)\right)^{-2 / n}=C_{n} .
\end{aligned}
$$

The last inequality follows from Lemma 2. 


$$
\left|n_{h}(\xi)\right| \leqslant \frac{1}{\gamma_{n}(h)}\left|\int_{0}^{\gamma_{n}(h)} e^{-2 \pi i \xi_{n} s} d s\right| \leqslant \frac{2}{\left|\xi_{n}\right| \gamma_{n}(h)}
$$

follows from van der Corput's lemma. Hence

$$
\begin{aligned}
\int_{2 a}^{N / 2}\left|n_{h}(\xi)\right|^{2} \frac{d h}{h} & \leqslant \frac{4}{\left|\xi_{n}\right|^{2}} \int_{2 a}^{N / 2} \frac{d h}{h\left[\gamma_{n}(h)\right]^{2}} \\
& \leqslant \frac{4}{\left|\xi_{n}\right|^{2}} \int_{2 a}^{N / 2} \frac{\gamma_{n}^{\prime}(h) d h}{\left[\gamma_{n}(h)\right]^{3}} \leqslant \frac{2}{\left[\left|\xi_{n}\right| \gamma_{n}(2 a)\right]^{2}}=2 .
\end{aligned}
$$

This completes the proof of (iv) and with it the proof of (i).

We have just proven (ii) in the case $p=2$. The case $p=\infty$ is obvious with $C_{n, \infty}=1$. The remaining case $2<p<\infty$ follows from these results and the Marcinkiewicz interpolation theorem. Q.E.D.

Since Theorem 3 is obvious for continuous functions, the estimate in Theorem 2(i) and a standard argument imply Theorem 3.

It is known that if $\gamma$ is a plane curve parametrized by arc length and the curvature of $\gamma$ is increasing, then $M$ is bounded on $L^{2}\left(\mathbf{R}^{2}\right)$. It is an interesting problem to determine similar geometric conditions on $\gamma$ in higher dimensions which would guarantee that $M$ is bounded on $L^{2}\left(\mathbf{R}^{n}\right)$.

\section{REFERENCES}

1. A. Nagel, N. M. Rivière and S. Wainger, On Hilbert transforms along curves. II, Amer. J. Math. 98 (1976), 395-403.

2. A A maximal function associated to the curve $\left(t, t^{2}\right)$, Proc. Nat. Acad. Sci. U.S.A. 73 (1976), 1416-1417.

3. A. Nagel, E. M. Stein and S. Wainger, Differentiation in lacunary directions, Proc. Nat. Acad. Sci. U.S.A. 75 (1978), 1060-1062.

4. A. Nagel and S. Wainger, Hilbert transforms associated with plane curves, Trans. Amer. Math. Soc. 223 (1976), 235-252.

5. E. M. Stein, Maximal functions: spherical means, Proc. Nat. Acad. Sci. U.S.A. 73 (1976), 2174-2175.

6. , Maximal functions: homogeneous curves, Proc. Nat. Acad. Sci. U.S.A. 73 (1976), 21762177.

7. E. M. Stein and S. Wainger, The estimation of an integral arising in multiplier transformations, Studia Math. 35 (1970), 101-104.

8. __ Maximal functions associated to smooth curves, Proc. Nat. Acad. Sci. U.S.A. 73 (1976), 4295-4296.

9. Problems in harmonic analysis related to curvature, Bull. Amer. Math. Soc. 84 (1978), 1239-1295.

10. S. Wainger, Applications of Fourier transforms to averages over lower dimensional sets, Proc. Sympos. Pure Math., vol. 35, Amer. Math. Soc., Providence, R.I., 1979.

11. A. Zygmund, Trigonometric series, vols. 1, 2, Cambridge Univ. Press, London, 1959.

Department of Mathematics and Statistics, University of South Carolina, Columbia, South Carolina 29208 